\title{
Agranulocitosis inducida por metimazol en pacientes con enfermedad de Graves
}

\author{
Methimazole-induced agranulocytosis in patients with Graves' disease \\ Helard Manrique-Hurtado ${ }^{1}$, Miguel Pinto-Valdivia ${ }^{2,3}$
}

\section{RESUMEN}

Objetivo: Describir las características clínicas y epidemiológicas de los pacientes con enfermedad de Graves que presentaron agranulocitosis inducida por metimazol. Material y métodos: Estudio retrospectivo, tipo serie de casos. Se revisaron las historias clínicas de todos los pacientes con diagnóstico de agranulocitosis inducida por metimazol, atendidos en el Hospital Nacional Arzobispo Loayza, entre enero 2002 y diciembre 2008. Se buscó asociación entre las variables demográficas y clínicas con la mortalidad y el tiempo de recuperación. Resultados: Treinta (0,60\%) pacientes con enfermedad de Graves fueron hospitalizados con el diagnóstico de agranulocitosis inducida por metimazol. La mediana de la edad fue 33,5 años y $86,67 \%$ fueron mujeres. $\mathrm{Al}$ ingreso, todos los pacientes presentaron fiebre y dolor de garganta. El manejo incluyó aislamiento invertido, suspensión del metimazol, administración de antibióticos y glucocorticoides. Doce (40\%) pacientes recibieron GM-CSF. El número de granulocitos se normalizó después de 10,59 días y cuatro $(13,33 \%)$ pacientes murieron por infecciones bacterianas y sepsis. En todos los casos, el tratamiento definitivo fue yodo radioactivo. No hubo diferencia significativa en la edad, sexo, dosis de metimazol, duración del tratamiento y uso de factor estimulante colonia, entre los pacientes fallecidos y los sobrevivientes. Además, el uso de factor estimulante de colonia no redujo el tiempo de recuperación de la agranulocitosis. Conclusión: La agranulocitosis inducida por metimazol es un evento adverso serio y potencialmente mortal. En este grupo de pacientes, la mortalidad fue elevada y el uso de factor estimulante de colonia no disminuyó el tiempo de recuperación.

PALABRAS CLAVE: Agranulocitosis, metimazol, antitiroideos, enfermedad de Graves, toxicidad medicamentosa. (Fuente: DeCS BIREME).

\section{SUMMARY}

Objective: To describe the clinical and epidemiological characteristics of patients with methimazole-induced agranulocytosis. Methods: A retrospective, case series study. We reviewed the medical records of all patients diagnosed with methimazole-induced agranulocytosis treated at Hospital Nacional Arzobispo Loayza between January 2002 and December 2008. We sought association between demographic and clinical variables with mortality and recovery time. Results: Thirty $(0.60 \%)$ patients with Graves' disease were hospitalized with a diagnosis of methimazole-induced agranulocytosis. The median age was 33,5 years and $86,67 \%$ were women. On admission, all patients had fever and sore throat. The treatment included isolation, suspension of methimazole, antibiotics and glucocorticoids. Twelve (40\%) patients received GM-CSF. The granulocyte count was normalized after 10,59 days and four (13.33\%) patients died from bacterial infections and sepsis. In all cases, the final treatment was radioiodine. There was no significant difference in age, sex, methimazole dose, duration of treatment, and use of stimulating factor, among the patients who died and survivors. Furthermore, the use of stimulating factor did not reduce the recovery time from agranulocytosis. Conclusion: Methimazole-induced agranulocytosis is a serious and potentially

\footnotetext{
Médico cirujano, especialista en Endocrinología, Servicio de Endocrinología, Hospital Nacional Arzobispo Loayza. Lima, Perú. Médico cirujano, especialista en Endocrinología, Servicio de Endocrinología, Hospital Nacional Cayetano Heredia. Lima, Perú. Facultad de Medicina Alberto Hurtado, Universidad Peruana Cayetano Heredia
} 
deadly adverse event. In this group of patients, mortality was high and the use of stimulating factor did not decrease the recovery time.

KEYWORDS: Agranulocytosis, methimazole, antithyroid agents, Graves' disease, drug toxicity. (Source: MeSH NLM).

\section{INTRODUCCIÓN}

Las tionamidas son las drogas de elección para el tratamiento del hipertiroidismo; especialmente, en mujeres jóvenes con diagnóstico de enfermedad de Graves (EG) (1). En nuestro país, sólo esta disponible el metimazol (MMI). Generalmente, es utilizado como terapia preparativa para la administración de yodo radioactivo. La dosis recomendada es de 15-30 $\mathrm{mg}$ por día; sin embargo, la enfermedad de muchos pacientes puede ser controlada con dosis menores (2).

Por otro lado, las tionamidas están asociadas a eventos adversos serios; como la agranulocitosis; se describe que ocurre en $0,35 \%$ de los pacientes que reciben MMI $(1,2)$. En la mayoría de estos pacientes, se presenta en los primeros 90 días de tratamiento $(1,3)$.

El objetivo del estudio fue describir las características clínicas y epidemiológicas de los pacientes con el diagnóstico de agranulocitosis inducida por metimazol.

\section{MATERIAL Y MÉTODOS}

Estudio descriptivo, retrospectivo, tipo serie de casos. Se revisaron las historias clínicas de todos los pacientes con diagnóstico de agranulocitosis inducida por metimazol (AIM), atendidos en el Hospital Nacional Arzobispo Loayza, entre enero 2002 y diciembre 2008.

Se registraron los datos de edad, sexo, diagnóstico previo de EG, duración de tratamiento con MMI, uso de factor estimulante de colonia (GM-CSF), tiempo de recuperación y mortalidad.

AIM fue definida como la cuenta de granulocitos menor de 500/ $\mu 1$ al momento del ingreso y con el antecedente de uso de MMI.

Los resultados fueron presentados como medidas de tendencia central y sus respectivas medidas de dispersión. Se buscó asociación entre las variables demográficas y clínicas con la mortalidad y el tiempo de recuperación, para lo cual se utilizó el programa STATA v. 8.0 (StataCorp LP, College Station, Texas). Se utilizó La prueba de U de Mann-Whitney para el análisis de las variables continuas y la prueba exacta de Fisher para las variables categóricas. Se consideró significativo una $\mathrm{p}<0,05$.

El estudio fue exonerado de revisión del Comité de Ética institucional, por ser un estudio de revisión de historias clínicas.

\section{RESULTADOS}

Entre enero 2002 y diciembre 2008, fueron atendidos 5151 pacientes con el diagnóstico de hipertiroidismo. De ellos, 4945 (86\%) tuvieron el diagnóstico de EG.

Treinta $(0,60 \%)$ pacientes con EG fueron hospitalizados con el diagnóstico de AIM. La mediana de la edad fue 33,5 años (rango intercuartil 28-43), 26 $(86,67 \%)$ fueron mujeres, el tiempo de presentación de síntomas antes del diagnóstico de EG fue 4,5 meses (rango intercuartil 3-23) y la duración del tratamiento con MMI previo al diagnóstico de AIM fue 3,5 meses (rango intercuartil 2-6). La frecuencia de AIM según dosis inicial de MMI se muestra en la tabla 1.

En todos los casos, el manejo incluyó aislamiento invertido, suspensión del MMI, administración de antibióticos y glucocorticoides. Doce $(40 \%)$ pacientes recibieron GM-CSF, y siete $(23,33 \%)$ recibieron carbonato de litio para controlar los síntomas de hipertiroidismo.

$\mathrm{Al}$ ingreso, todos los pacientes presentaron fiebre y dolor de garganta. El número de granulocitos se normalizó después de 10,59 $\pm 5,43$ días. Cuatro $(13,33 \%)$ pacientes murieron por infecciones bacterianas y sepsis. En todos los casos, el tratamiento definitivo fue yodo radioactivo, en 13 (43,33\%) pacientes fue administrado durante la hospitalización.

No hubo diferencia significativa en la edad, sexo, dosis de MMI, duración del tratamiento y uso de factor estimulante colonia, entre los pacientes fallecidos y los 
Tabla 1. Agranulocitosis inducida por metimazol (MMI), según dosis inicial recibida $(n=30)$.

\begin{tabular}{ccc}
\hline Dosis inicial de MMI & n & \% \\
\hline $15 \mathrm{mg} /$ día & 2 & 6,67 \\
$30 \mathrm{mg} /$ día & 17 & 56,67 \\
$40 \mathrm{mg} /$ día & 6 & 20,00 \\
$60 \mathrm{mg} /$ día & 5 & 16,67 \\
\hline
\end{tabular}

sobrevivientes. Además, el uso de factor estimulante de colonia no redujo el tiempo de recuperación de la $\operatorname{AIM}(\mathrm{p}=0,53)$.

\section{DISCUSIÓN}

La mayoría de efectos adversos asociados a las tionamidas son leves y transitorios. En 5\% de los pacientes, se puede presentar rash y prurito (2).

El efecto adverso más temido es la agranulocitosis de causa autoinmune (1), que se asocia con elevada mortalidad. En un estudio de vigilancia farmacológica, la agranulocitosis y neutropenia, fueron las causas más frecuentes (49\%) de muerte asociada al uso de estas drogas (4). Por otro lado, la reactividad cruzada entre el MMI y el propiltiouracilo (PTU) para inducir agranulocitosis está bien documentada, por lo que no se recomienda intercambiar tionamidas (1).

La mayoría de casos de AIM se presentan en los primeros 90 días; sin embargo, esta complicación se puede presentar en cualquier momento del tratamiento (1-4).

En estudios previos, la agranulocitosis se presentó entre 2-12 semanas después de iniciado el tratamiento $(5,6)$. En un reciente estudio retrospectivo, que incluyó 50385 pacientes con EG, esta complicación se presentó en 55 pacientes después de 69 días de tratamiento (rango 11-233 días) (7). En nuestro estudio, la AIM se presentó después de 3,5 meses de tratamiento; pero, algunos pacientes la presentaron después de 6 meses.

No hay evidencia concluyente acerca de, que la AIM podría ser dosis dependiente. En un estudio retrospectivo de casi 6000 pacientes, se encontró mayor frecuencia de AIM en los pacientes que recibieron MMI $30 \mathrm{mg} /$ día, en comparación con los que recibieron MMI $15 \mathrm{mg} /$ día $(0,814 \%$ vs. $0,219 \%$, $p<0,01$ ) (8). Otro estudio encontró que la AIM fue 11 veces más frecuente en el grupo que recibió $30 \mathrm{mg} / \mathrm{día}$ (6).

Los pacientes que reciben MMI $40 \mathrm{mg}$ /día tienen 8,6 veces mayor riesgo de AIM, en comparación con los que reciben dosis menores (9). Por otro lado, las reacciones adversas son más frecuentes con dosis mayores de MMI (10).

En nuestro estudio, la mayoría de pacientes recibieron MMI 30-40 mg/día. No se recolectó la dosis inicial de MMI de todos los pacientes, por lo que no se puede determinar la frecuencia de AIM de acuerdo a la dosis inicial. Se debe resaltar que $36,67 \%$ de los pacientes recibieron dosis de MMI (40-60 mg/día) por encima de lo recomendado. Es importante recalcar que la mayoría de estudios reportaron eficacia similar entre la dosis de MMI $15 \mathrm{mg} /$ día y $30 \mathrm{mg} /$ día, para normalizar los niveles de T4 libre (2).

Todos los pacientes acudieron por presentar fiebre y dolor de garganta. En nuestro país, no es práctica usual hacer monitoreo seriado de la cuenta leucocitaria. Generalmente, se solicita un hemograma antes de iniciar el tratamiento con MMI.

En un estudio realizado en 15398 pacientes con EG, se presentaron 55 casos de AIM; de ellos, sólo 21,82\% fueron diagnosticados por la presencia de fiebre y dolor de garganta; $78,18 \%$ fueron detectados durante el monitoreo de la cuenta leucocitaria (sin síntomas de infección) (11). De manera similar, en una serie de casos de 109 pacientes con AIM, 18(16,5\%) tuvieron $>3000$ leucocitos $/ \mathrm{mm}^{3}$ al inicio del tratamiento. En el seguimiento, 4 casos se detectaron por síntomas de infección, 10 presentaron disminución de la cuenta leucocitaria y 4 se detectaron a través del monitoreo del hemograma. Los autores concluyeron que un factor de riesgo para AIM, fue la cuenta leucocitaria basal de 3000 - 3900 leucocitos por $\mathrm{mm}^{3}$ (3). 
Los pacientes con EG, ocasionalmente pueden cursar con neutropenia antes de iniciar el tratamiento con tionamidas $(1,6)$ y la agranulocitosis es de naturaleza impredecible e idiosincrática (2), por esta razón, la mayoría de expertos no recomienda el monitoreo del hemograma (2). Es importante educar al paciente acerca de esta complicación, indicando suspender el uso de MMI si presenta fiebre y dolor de garganta.

El pronóstico de la AIM ha mejorado con los avances en la terapia de soporte, que incluye antibióticos, transfusiones sanguíneas y el uso de GMCSF (7).

La administración de factor estimulante podría reducir el tiempo de recuperación y la estancia hospitalaria (1). En un estudio retrospectivo de 30798 pacientes con EG, el uso de GM-CSF acortó el tiempo de recuperación de 9,2 a 5,5 días, $\mathrm{p}<0,01$ (12). Además, el factor estimulante de colonia normalizó más rápidamente la temperatura corporal (5). A pesar que un estudio prospectivo, no mostró diferencia en el tiempo de recuperación de la AIM (13), la mayoría de expertos recomiendan su uso (1).

Nuestro estudio tiene varias limitaciones. En primer lugar, el hecho que sea un estudio retrospectivo, de revisión de historias clínicas, no nos permite asegurar que toda la información relevante haya sido consignada de manera adecuada en las historias clínicas (por ejemplo, dosis diaria y número de días de tratamiento con factor estimulante de colonia). Por otro lado, existe la posibilidad de que no todos los casos de AIM hayan sido consignados (historias clínicas extraviadas, depuradas, o incompletas). Sin embargo, una fortaleza es el número de pacientes evaluados ( $>$ 5 000) y el período de estudio (7 años) en un hospital de referencia, donde se atienden mayormente mujeres. El uso de varias fuentes de información (libro de interconsultas del Servicio de Endocrinología, hojas de Registro Diario de Atención y otras actividades de salud de los consultorios y hojas de epicrisis de las salas de hospitalización) nos ha permitido reducir el número de historias clínicas no evaluadas.

En conclusión, la AIM es un evento serio y potencialmente mortal, posiblemente dosisdependiente. En este grupo de pacientes, la mortalidad fue elevada y el uso de factor estimulante de colonia no disminuyó el tiempo de recuperación.

\section{Declaración de Financiamiento y de conflictos de intereses:}

El estudio fue financiado por los autores. Los autores declaran no tener conflictos de intereses.

\section{Correspondencia:}

Miguel Pinto Valdivia

Servicio de Endocrinología, Hospital Nacional

Cayetano Heredia (Lima, Perú)

Av. Honorio Delgado 262, Lima 31, Perú

Teléfono: +511992706110

Fax: +5114814177

Email: miguelpinto72@yahoo.com

\section{REFERENCIAS BIBLIOGRÁFICAS}

1. Cooper DS. Antithyroid drugs. N Engl J Med. 2005; 352: 905-17.

2. Franklyn JA. Thyroid gland: Antithyroid therapybest choice of drug and dose. Nat Rev Endocrinol. 2009; 5: 592-4.

3. Tajiri J, Noguchi S. Antithyroid drug-induced agranulocytosis: special reference to normal white blood cell count agranulocytosis. Thyroid. 2004; 14:459-62.

4. Pearce SH. Spontaneous reporting of adverse reactions to carbimazole and propylthiouracil in the UK. Clin Endocrinol (Oxf). 2004; 61:589-94.

5. Dai WX, Zhang JD, Zhan SW, et al. Retrospective analysis of 18 cases of Antithyroid drug (ATD). induced agranulocytosis. Endocr J. 2002; 49:29-33.

6. Tsuboi K, Ueshiba H, Shimojo M, et al. The relation of initial methimazole dose to the incidence of methimazole-induced agranulocytosis in patients with Graves' disease. Endocr J. 2007; 54:39-43.

7. Watanabe N, Narimatsu H, Noh JY, et al. Antithyroid drug-induced hematopoietic damage: A retrospective cohort study of agranulocytosis and pancytopenia involving 50385 patients with Graves' disease. J Clin Endocrinol Metab. 2012; 97:E49-E53.

8. Takata K, Kubota S, Fukata S, et al. Methimazoleinduced agranulocytosis in patients with Graves' disease is more frequent with an initial dose of 30 $\mathrm{mg}$ daily than with $15 \mathrm{mg}$ daily. Thyroid. 2009; 19:559-63.

9. Cooper DS, Goldminz D, Levin AA, et al. Agranulocytosis associated with antithyroid drugs. Ann Intern Med. 1983; 98: 26-29.

10. Reinwein D, Benker $\mathrm{G}$, Lazarus $\mathrm{JH}$, et al. A prospective randomized trial of antithyroid drug dose in Graves' disease therapy. J Clin Endocrinol 
Metab. 1993; 76:1516-1521.

11. Tajiri J, Noguchi S, Murakami T, Murakami N. Antithyroid drug-induced agranulocytosis. The usefulness of routine white blood cell count monitoring. Arch Intern Med. 1990; 150:621-4.

12. Tajiri J, Noguchi S. Antithyroid drug-induced agranulocytosis: how has granulocyte colonystimulating factor changed therapy? Thyroid. 2005; $15: 292-7$.
13. Fukata S, Kuma K, Sugawara M. Granulocyte colony-stimulating factor (G-CSF) does not improve recovery from antithyroid drug-induced agranulocytosis: a prospective study. Thyroid. 1999; 9:29-31.

Recibido: 11/12/2012

Aceptado: 16/04/2013 